\title{
OTIMIZAÇÃO DO PLANEJAMENTO SEMANAL DAS MÁQUINAS DE LINGOTAMENTO CONTÍNUO DA ARCELORMITTAL TUBARÃO *
}

Marcos Fernando Campioto Junior ${ }^{1}$ Alerio de Brito Silva²

\begin{abstract}
Resumo
O objetivo deste trabalho é apresentar os resultados obtidos, após a implantação da ferramenta de otimização do planejamento e programação da produção CScheduler Weekly Planner da PSI Metals na ArcelorMittal Tubarão. A ferramenta de otimização CScheduler permite realizar o planejamento e programação de médio prazo para as máquinas de lingotamento contínuo da ArcelorMittal Tubarão, visando melhor atendimento as regras de negócio relacionadas a cliente, redução custos operacionais e redução de instabilidade operacional do processo. Este trabalho também apresenta a criação de um novo Modus Operandi para o planejamento e programação com resultados obtidos em casos de testes e resultados pósimplantação em produção.
\end{abstract}

Palavras-chave: Planejamento; Programação da Produção; Otimização; Lingotamento Contínuo.

\section{OPTIMIZATION OF CONTINUOUS CASTING WEEKLY PLANNING AT ARCELORMITTAL TUBARÃO}

\begin{abstract}
The objective of this work is to present the results obtained after the implementation of the planning and scheduling optimization tool of the CScheduler Weekly Planner from PSI Metals at ArcelorMittal Tubarão. The CScheduler optimization tool enables the medium-term planning and scheduling of ArcelorMittal Tubarão continuous casting machines, aiming at better compliance with customer-related business rules, reducing operating costs and reducing operational instability in the process. This work also presents the creation of a new Modus Operandi for the planning and programming with results obtained in cases of tests and results post-implantation in production.
\end{abstract}

Keywords: Planning; Production Scheduling; Optimization; Continuous casting.

1 Bacharelado em Sistemas de Informação, Pós-Graduado em Engenharia de Produção, Especialista em Desenho Integrado de Processos, Gerência de Desenho Integrado de Processos, ArcelorMittal Tubarão, Vitória, ES, Brasil.

2 Bacharelado em Engenharia Metalúrgica, Técnico Especializado em Programação e Controle da Produção, Gerência de Área de Programação e Controle Industrial, ArcelorMittal Tubarão, Vitória, ES, Brasil. 


\section{INTRODUÇÃO}

A ArcelorMittal Tubarão tem como foco a produção de produtos de aços planos semi-acabados (placas e bobinas de aço). As placas são fabricadas pelas unidades de Lingotamento Contínuo da Aciaria da ArcelorMittal Tubarão. Com o aumento da variabilidade da carteira de pedidos de placa venda para laminação, é necessária uma evolução nos processos de planejamento e programação da produção para garantir 0 atendimento aos pedidos sem afetar a estabilidade operacional da Aciaria. Além disso, é necessário utilizar mais tempo para o planejamento, do que para a execução dos processos de programação.

"O propósito do planejamento e controle é garantir que os processos de produção ocorram eficaz e eficientemente e que produzam produtos e serviços conforme requeridos pelos consumidores" (Slack et al., 2002, p. 314) [1]. Lembrando que, a entrega on time de produtos acabados é essencial para satisfação do cliente, e este é um fator crítico para sobrevivência do negócio de hoje em mercados altamente competitivos (GUO et al., 2006) [2].

As grandes corporações que visam a manutenção da competitividade no mercado que atuam, devem se desdobrar para manter um plano tecnológico atualizado. Segundo Linn et al. (2000) [3] Tecnologia de gestão inclui: (1) o planejamento para o desenvolvimento das capacidades tecnológicas; (2) identificar tecnologias-chave e suas áreas afins para o desenvolvimento; (3) a determinação de "comprar" ou "fazer", ou seja, se a importação ou a auto-desenvolvimento deve ser perseguido, e (4) a criação de mecanismos institucionais para dirigir e coordenar o desenvolvimento das capacidades tecnológicas, bem como a elaboração de medidas de política de controles.

Para isto é necessário a adoção de novas estratégias de crescimento e estratégias operacionais para manter a estabilidade operacional de seus equipamentos e unidades produtivas. De acordo com Slack e Lewis (2009, p. 27) [4] "as empresas acreditam que a forma como elas gerenciam suas operações é que as distingue e as coloca acima de seus concorrentes".

Não obstante aos processos tecnológicos atualizados, as grandes corporações também tem que se preocupar com o aumento da produtividade do homem, para com isto, aumentar a lucratividade e retorno financeiro. Para Gaither e Frazier (2001) [5] a essência das estratégias de operações é a formação de estratégias de posicionamento (produtos personalizados ou padronizados, produção focalizada no produto ou no processo, e estoques baseados na produção para estoque ou produção sob encomenda).

Estas estratégias demandam novas soluções de Sistemas de Planejamento e Programação da Produção, aderentes às necessidades atuais e facilmente moldáveis às novas demandas. Uma empresa moderna com alta competitividade no mercado possui um sistema flexível de produção, com rapidez no projeto e implementação de novos produtos, com baixo tempo de aprovisionamento e estoques reduzidos que atendam a necessidades dos clientes (Tubino, 2000 [6]). Desta forma o planejamento, programação e controle da produção têm papel fundamental no sucesso organizacional. 
O objetivo deste trabalho é apresentar os resultados obtidos, após a implantação da ferramenta de otimização do planejamento e programação da produção CScheduler Weekly Planner da PSI Metals na ArcelorMittal Tubarão. A ferramenta tem como objetivo criar um planejamento de médio prazo de Lingotamento Contínuo visando melhor atendimento aos grupos de pedidos, redução custos operacionais e redução de instabilidade do processo. A ferramenta cria um cenário de programação de médio prazo atendendo ao objetivo geral configurados na ferramenta.

\section{MATERIAIS E MÉTODOS}

Para viabilizar o atendimento dos objetivos esperados, foi necessário realizar uma série de parametrizações, configurações e ajustes na ferramenta de otimização, inclusive alteração do modus operandi da programação da produção.

\section{1 - Revisão do Modus Operandi da Programação da Produção}

Foi realizada a revisão do modus operandi do processo de programação (figura 1) do Lingotamento Contínuo, para permitir que o planejamento da carteira seja feito dentro da ferramenta de otimização. Antes o planejamento era feito totalmente fora da ferramenta de otimização e diariamente era feita a programação manual da carteira. Após a implantação do CScheduler WP, o planejamento passou a ser realizado dentro da ferramenta de otimização.

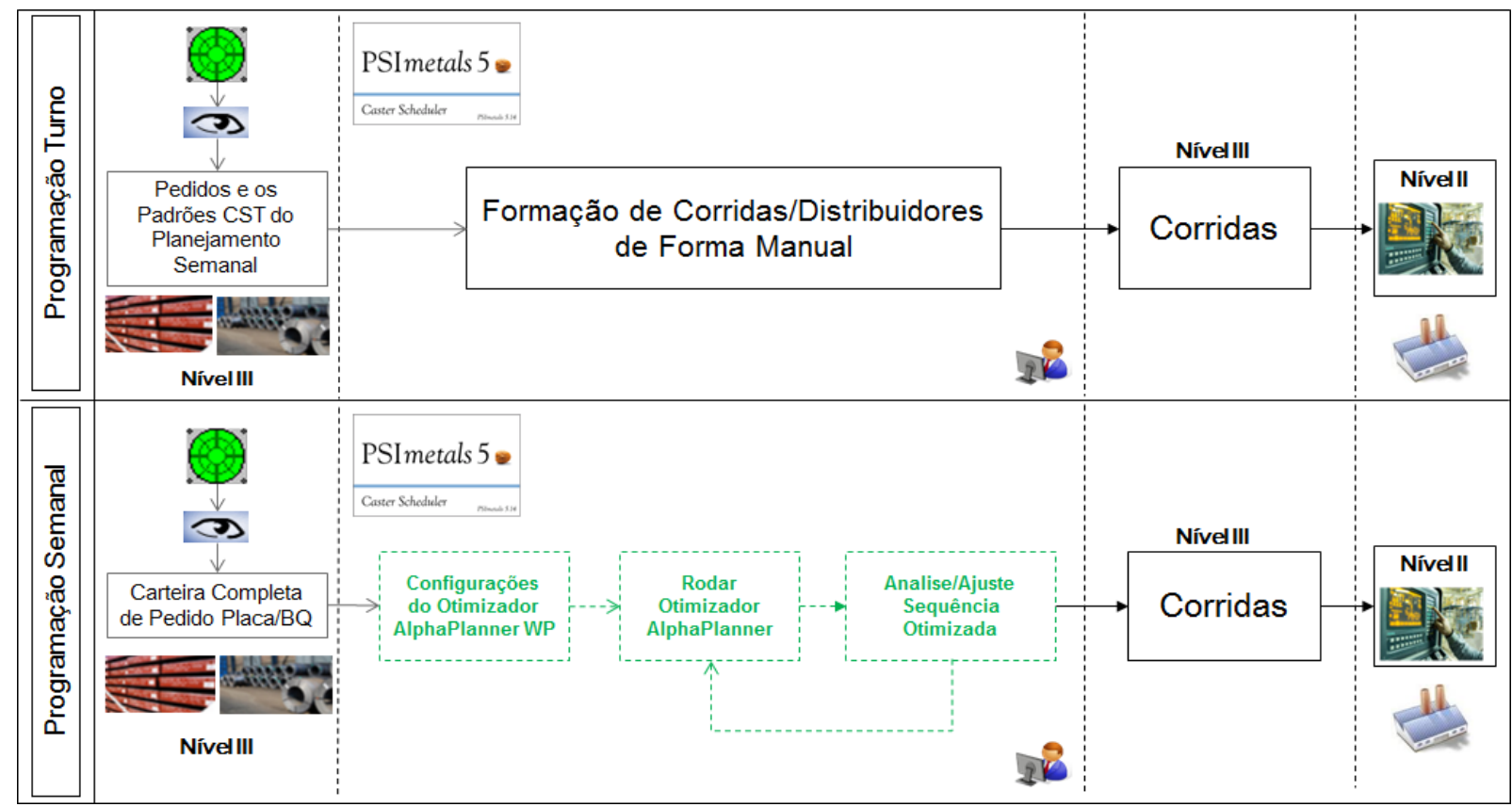

Figura 1. Modus Operandi de Planejamento e Programação

As parametrizações, configurações e ajustes realizados na ferramenta, seguiram uma ordem de prioridade, sempre levando em consideração como regra principal às restrições dos equipamentos de lingotamento contínuo. As regras de programação padronizadas, foram analisadas criteriosamente e as regras de negócio foram detalhadas para permitir a programação semanal com o uso da ferramenta de otimização. 


\section{2 - Premissas, Parametrizações e Tuning no sistema CScheduler WP}

A. A ferramenta deve atender à programação por períodos pré-estabelecidos na Configuração da Sequência. Esta configuração é necessária para limitar o volume a ser utilizado na programação da carteira completa de pedidos. $\mathrm{O}$ período de programação deverá estar alinhado com o volume disponível em carteira.

A1. A ferramenta de otimização deve cumprir com os padrões de programação da Aciaria (Ex. recorte nas dimensões da placa requeridas pelo pedido, saltos de largura permitidos, peso mínimo e máximo de cada corrida e demais regras implementadas atualmente).

A2. Os itens "B" a "l" são parâmetros de tuning e são apresentados abaixo em ordem de prioridade. Tais parâmetros podem ser conflitantes (trade offs) e representam metas a serem buscadas pela ferramenta de otimização. Há necessidade, sempre, de se observar o resultado global. A maioria dos itens depende do mix da carteira de pedidos e a validação do resultado deve ser ponderada com cada business case (BC5, BC6 e BC7).

B. Atendimento aos grupos de pedidos do cockpit

$\rightarrow$ Desejável programação de $100 \%$ dos pedidos itens com formação de lote tanto para padrão de aço quanto para largura/obrigatoriedade de MLC, podendo:

- Fazer misturas de Padrões de Aço no mesmo distribuidor;

- Antecipar pedidos de outras categorias;

- Gerar passivo na carteira;

- Programar Mudança de Largura (ML);

- Impactar Ritmo Diário;

- Gerar Excesso.

- Não Desejável:

- Programação de Corrida Solteira (Distribuidor com uma ou duas corridas).

- Não pode:

- Descumprir o Padrão de Programação da Aciaria.

- $\quad$ Cockpit = Pedidos identificados com "S" na coluna Cockpit

$\Rightarrow$ Os pedidos deste grupo devem ter todas as placas do pedido-item programadas, sem exceção;

$\Rightarrow$ Os pedidos deste grupo devem ser concentrados no início da semana programada;

- Definição de Formação de lote: o pedido item juntamente com demais itens do mesmo padrão de aço na carteira de pedidos deve ter disponível para programação acima de 810 toneladas (tolerância superior dos pedidos) devendo o volume ser produzível no mesmo distribuidor com saltos de largura dentro do padrão. O volume disponível para programação poderá ter saldo abaixo de 810 toneladas desde que seja possível misturá-lo com outro Padrão de Aço obtendo número de corridas no distribuidor maior ou igual a três corridas.

C. Atendimento aos pedidos prioritários/críticos

$\rightarrow$ Desejável programação de $95 \%$ dos pedidos itens com formação de lote tanto para padrão de aço quanto para largura/obrigatoriedade de MLC, podendo: 
- Fazer misturas de Padrões de Aço no mesmo distribuidor;

- Antecipar pedidos de outras categorias;

- Gerar passivo na carteira;

- Programar ML.

- Impactar Ritmo Diário;

- Não Desejável:

- Gerar Excesso;

- Programar Corrida Solteira.

- Não pode:

- Descumprir o Padrão de Programação da Aciaria.

- Pedidos identificados como prioritários"

- Definição de Formação de lote: o pedido item juntamente com demais itens do mesmo padrão de aço na carteira de pedidos deve ter disponível para programação acima de 810 toneladas (tolerância superior dos pedidos) devendo o volume ser produzível no mesmo distribuidor com saltos de largura dentro do padrão. O volume disponível para programação poderá ter saldo abaixo de 810 toneladas desde que seja possível misturá-lo com outro padrão de aço obtendo número de corridas no distribuidor maior ou igual a três corridas/panelas de aço.

D. Atendimento aos ritmos/restrições de médio prazo

$\rightarrow$ Desejável atendimento do ritmo de médio prazo dentro da faixa estipulada, podendo:

- Fazer misturas de Padrões de Aço no mesmo distribuidor;

- Antecipar pedidos de outros grupos/categorias;

- Gerar passivo na carteira;

- Programar ML.

- Não Desejável:

- Programar Corrida Solteira;

- Gerar Excesso.

- Não pode:

- Descumprir o Padrão de Programação da Aciaria.

E. Desejável tamanho médio de distribuidor $>=8$ corridas/distribuidor

- Fazer misturas de Padrões de Aço no mesmo distribuidor;

- Antecipar pedidos de outros grupos/categorias;

- Programar ML.

- Não Desejável:

- Gerar passivo na carteira;

- Impactar Ritmo Diário;

- Gerar Excesso.

- Não pode:

- Descumprir o Padrão de Programação da Aciaria.

F. Atendimento à data limite de vazamento:

Material Passivo até 4 dias = Data de Programação -4 dias<Data Limite de Vazamento < Data de Programação

Material Prazo $=$ Data de Programação <= Data Limite de Vazamento <= Data de Programação $+5 d$ 
Material Passivo há mais de 4 dias $=$ Data Limite de Vazamento $<$ Data de Programação - 4 dias

$\underline{\text { Material Antecipado }}=$ Data Limite de Vazamento $>$ Data de Programação $+5 d$

Criar classificação de prioridades, sendo: mais prioritário é o passivo até quatro dias, segundo prioritário é o material do prazo, terceiro prioritário é o passivo há mais de quatro dias e o menos prioritário é o antecipado.

Desejável atendimento da data limite de vazamento, podendo:

- Fazer misturas de Padrões de Aço no mesmo distribuidor;

- Antecipar pedidos de outros grupos/categorias;

- Programar ML.

- Não Desejável:

- Impactar Ritmo Diário;

- Gerar Excesso;

- Programar Corrida Solteira.

- Não pode:

- Descumprir o Padrão de Programação da Aciaria.

G. Excesso

$\rightarrow$ Desejável o menor volume de "overage" programado possível, sendo desejável no máximo $2 \%$ de "overage" no volume programado para pedidos de produto BQ. Esse item essencialmente depende do mix da carteira de pedidos e validação do resultado deve ser ponderada em cada business case.

$\mathrm{H}$. Mudança de largura (ML)

$\rightarrow$ Desejável programação de $\mathrm{ML}<=36$ placas/dia:

- Pode:

- Fazer misturas de Padrões de Aço no mesmo distribuidor;

- Antecipar pedidos de outros grupos/categorias;

- Gerar passivo na carteira.

- Não Desejável:

- Troca de largura fechando nas três primeiras placas do distribuidor nos dois veios;

- Impactar Ritmo Diário;

- Gerar Excesso.

- Não pode:

- Descumprir o Padrão de Programação da Aciaria.

I. Mistura de Padrões de Aço no mesmo distribuidor

$\rightarrow$ É desejável que a quantidade de misturas seja feita de maneira controlada, sendo explorada como recurso para atender aos itens acima ("A" à "G")

- Pode:

- Fazer misturas de Padrões de Aço no mesmo distribuidor;

- Antecipar pedidos de outros grupos/categorias.

- Não Desejável:

- Programar ML;

- Programar Corrida Solteira;

- Impactar Ritmo Diário;

- Gerar Excesso. 
- Não pode:

- Descumprir o Padrão de Programação da Aciaria.

\section{RESULTADOS E DISCUSSÃO}

Os motivadores deste projeto foram: a) redução do desvio médio de placas para corte por mudança de largura (depende do mercado e/ou mix); b) redução do passivo de carteira de pedidos; c) aumento do sequencial de corridas por distribuidor; d) redução de corridas solteiras com a liberação de misturas de Padrões de Aço no mesmo distribuidor.

Após os ajustes, parametrização e tuning, foram realizadas três simulações com dados reais de produção em carteiras completas de pedidos. Desta forma, utilizando a ferramenta, foi possível obter os resultados abaixo detalhados nas tabelas (tabela 1,2 e 3) e figuras (figuras 2, 3 e 4).

Tabela 1. Comparação dos resultados com uso da ferramenta de otimização - Case 1

\begin{tabular}{|c|c|c|c|}
\hline SEQUENCE EVALUATION & Manual & Automática & $\begin{array}{c}\text { Automática c/ } \\
\text { Ajustes }\end{array}$ \\
\hline Number of tundishes & 35 & 33 & 32 \\
\hline AVERAGE Heats/Tundishes Total & 11.43 & 13.12 & 13.09 \\
\hline AVERAGE Heats/Tundishes MLC-1 & 11.54 & 12.77 & 12.54 \\
\hline AVERAGE Heats/Tundishes MLC-2 & 11.50 & 13.30 & 13.56 \\
\hline AVERAGE Heats/Tundishes MLC-3 & 11.20 & 13.40 & 13.40 \\
\hline Number of heats & 400 & 433 & 419 \\
\hline Number of width changes up & 51 & 50 & 42 \\
\hline Number of width changes up MLC-1 & 24 & 18 & 20 \\
\hline Number of width changes up MLC-2 & 21 & 29 & 19 \\
\hline Number of width changes up MLC-3 & 6 & 3 & 3 \\
\hline Number of width changes down & 47 & 48 & 42 \\
\hline Number of width changes down MLC-1 & 21 & 20 & 18 \\
\hline Number of width changes down MLC-2 & 23 & 24 & 20 \\
\hline Number of width changes down MLC-3 & 3 & 4 & 4 \\
\hline Number of EQUAL changes & 5 & 11 & 8 \\
\hline Number of LIKE changes & 2 & 10 & 8 \\
\hline Number of MIX changes & 1 & 11 & 9 \\
\hline Number of MIX1 changes & 2 & 8 & 3 \\
\hline Number of MIX2 changes & 2 & 3 & 2 \\
\hline Number of SCRAP changes & 0 & 0 & 0 \\
\hline
\end{tabular}




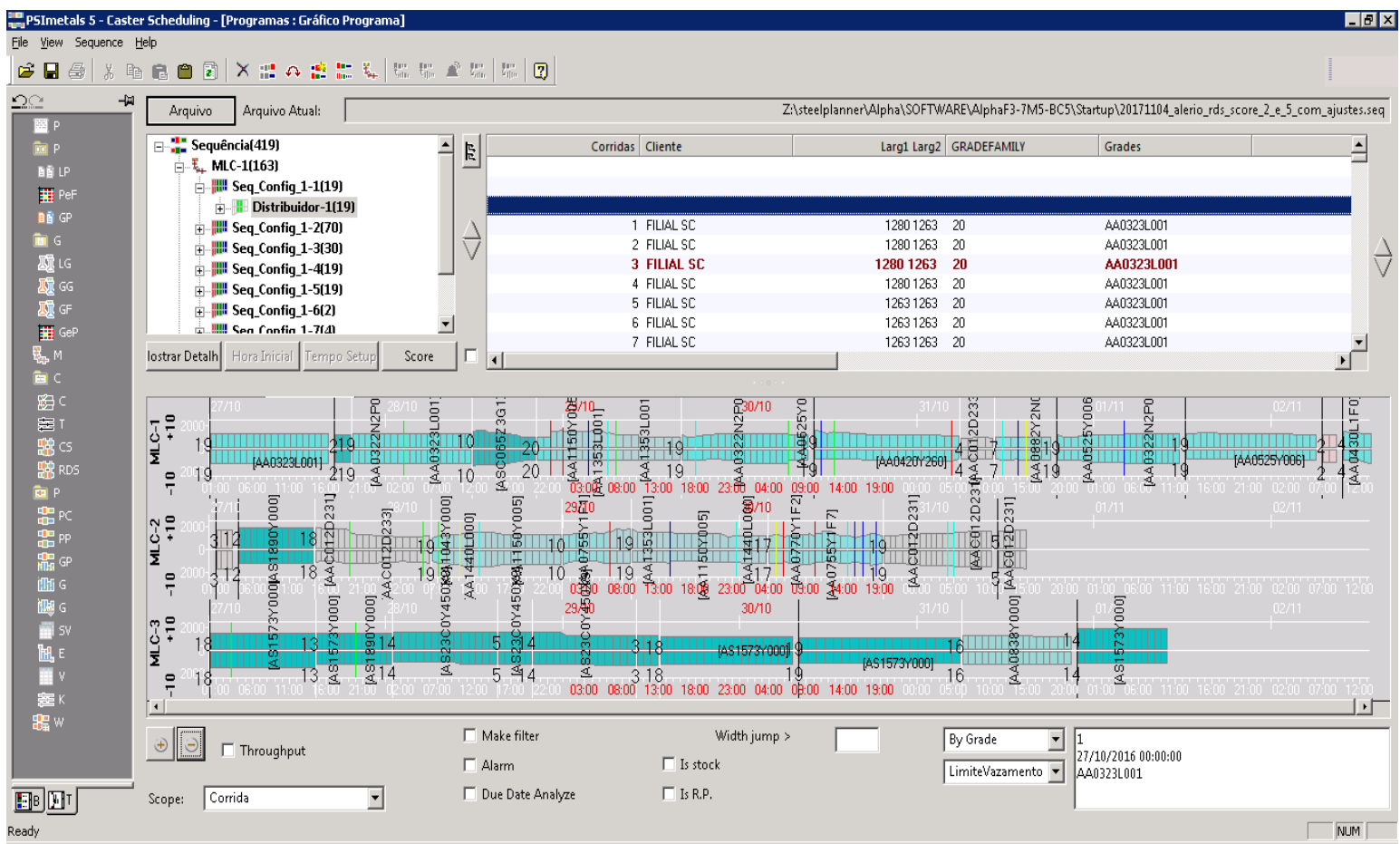

Figura 2. Resultado gráfico do planejamento e programação semanal - Case 1

Tabela 2. Comparação dos resultados com uso da ferramenta de otimização - Case 2

SEQUENCE EVALUATION Manual Automática Automática c/

\begin{tabular}{lccc}
\hline Number of tundishes & 28 & 27 & 28 \\
\hline AVERAGE Heats/Tundishes Total & 12.82 & 15.30 & 13.79 \\
\hline AVERAGE Heats/Tundishes MLC-1 & 12.25 & 12.29 & 9.56 \\
\hline AVERAGE Heats/Tundishes MLC-2 & 11.60 & 15.73 & 14.60 \\
\hline AVERAGE Heats/Tundishes MLC-3 & 14.50 & 17.11 & 17.11 \\
\hline Number of heats & 359 & 413 & 386 \\
\hline Number of width changes up & 30 & 26 & 25 \\
\hline Number of width changes up MLC-1 & 14 & 7 & 9 \\
\hline Number of width changes up MLC-2 & 15 & 19 & 16 \\
\hline Number of width changes up MLC-3 & 1 & 0 & 0 \\
\hline Number of width changes down & 31 & 33 & 13 \\
\hline Number of width changes down MLC-1 & 11 & 16 & 15 \\
\hline Number of width changes down MLC-2 & 20 & 17 & 0 \\
\hline Number of width changes down MLC-3 & 0 & 3 & 6 \\
\hline Number of EQUAL changes & 6 & 9 & 4 \\
\hline Number of LIKE changes & 3 & 7 & 5 \\
\hline Number of MIX changes & 1 & 9 & 3 \\
\hline Number of MIX1 changes & 0 & 4 & 4 \\
\hline Number of MIX2 changes & 0 & 5 & 1 \\
\hline Number of SCRAP changes & 0 & 1 &
\end{tabular}




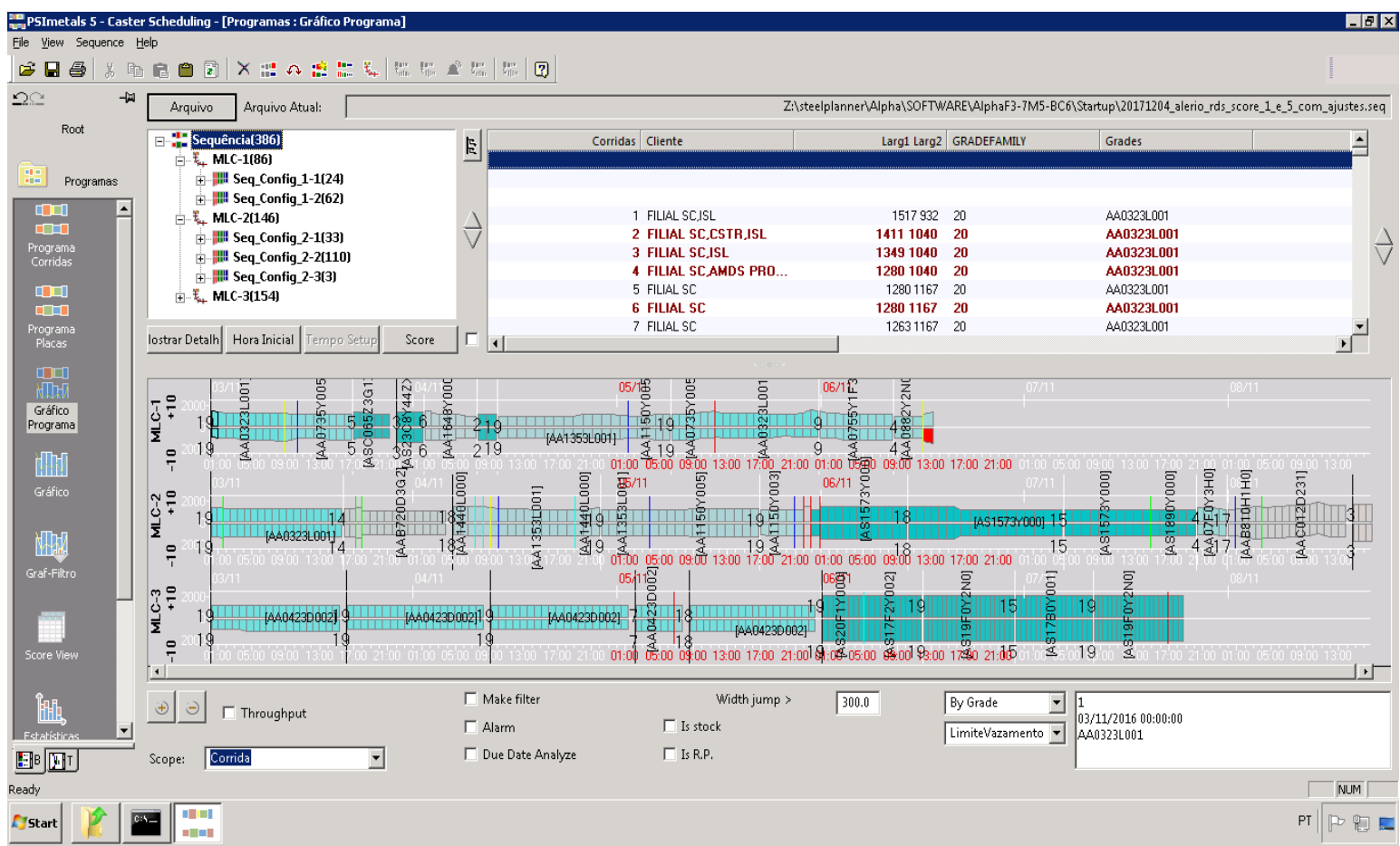

Figura 3. Resultado gráfico do planejamento e programação semanal - Case 2

Tabela 3. Comparação dos resultados com uso da ferramenta de otimização - Case 3

SEQUENCE EVALUATION Manual Automática Automática c/

\begin{tabular}{lccc} 
& & Automatica & Ajustes \\
\hline Number of tundishes & 39 & 35 & 35 \\
\hline AVERAGE Heats/Tundishes Total & 10.08 & 13 & 12.71 \\
\hline AVERAGE Heats/Tundishes MLC-1 & 12.73 & 12.50 & 13.00 \\
\hline AVERAGE Heats/Tundishes MLC-2 & 9.73 & 15.73 & 15.00 \\
\hline AVERAGE Heats/Tundishes MLC-3 & 8.23 & 9.92 & 9.83 \\
\hline Number of heats & 393 & 455 & 445 \\
\hline Number of width changes up & 34 & 33 & 28 \\
\hline Number of width changes up MLC-1 & 9 & 8 & 9 \\
\hline Number of width changes up MLC-2 & 24 & 23 & 17 \\
\hline Number of width changes up MLC-3 & 1 & 2 & 2 \\
\hline Number of width changes down & 40 & 48 & 45 \\
\hline Number of width changes down MLC-1 & 14 & 16 & 21 \\
\hline Number of width changes down MLC-2 & 24 & 30 & 22 \\
\hline Number of width changes down MLC-3 & 2 & 2 & 2 \\
\hline Number of EQUAL changes & 2 & 4 & 9 \\
\hline Number of LIKE changes & 1 & 8 & 2 \\
\hline Number of MIX changes & 1 & 4 & 2 \\
\hline Number of MIX1 changes & 3 & 2 & 2 \\
\hline Number of MIX2 changes & 1 & 0 & 0 \\
\hline Number of SCRAP changes & 0 & 0 & \\
\hline
\end{tabular}




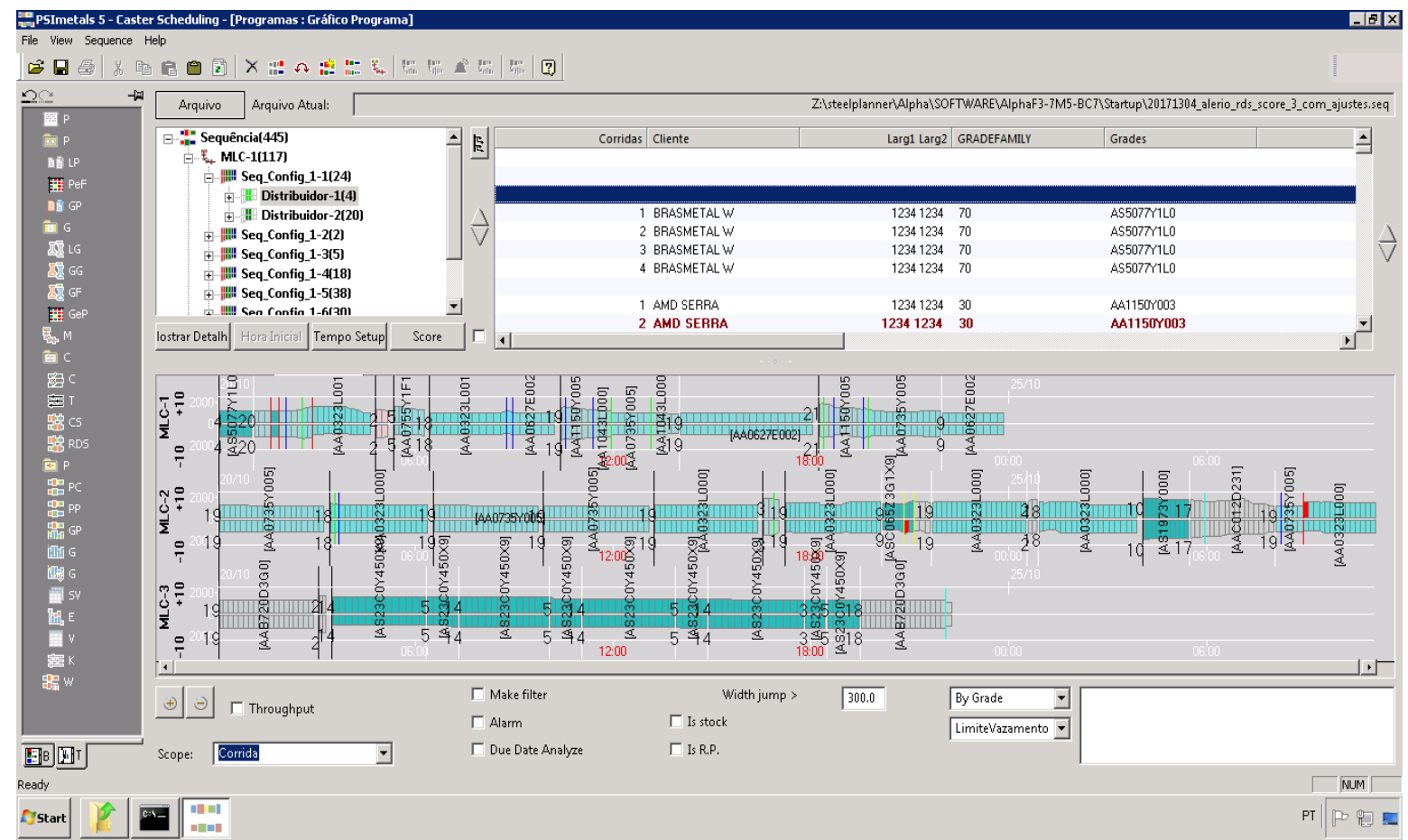

Figura 4. Resultado gráfico do planejamento e programação semanal - Case 2

Os resultados apresentados nas tabelas 1, 2 e 3 confirmar que o uso da ferramenta de otimização para o planejamento e programação das máquinas de lingotamento contínuo, otimizam o processo de forma global.

Após a implantação da ferramenta de otimização CScheduler WP, os resultados continuaram aderentes aos resultados obtidos nos casos de testes, conforme apresentado na figura 5 .

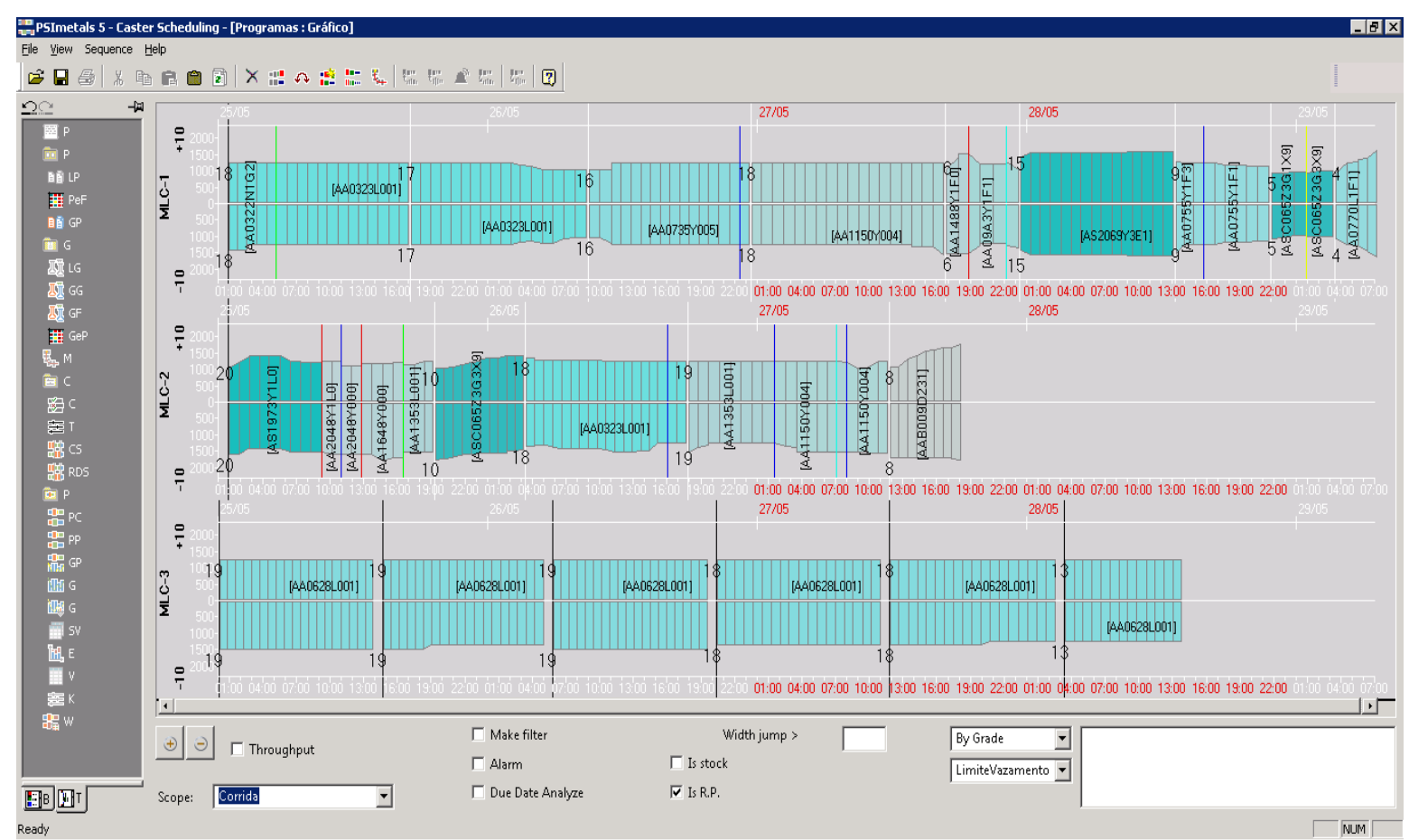

Figura 5. Resultado da Programação das MLC's em Produção 


\section{CONCLUSÃO}

Após realizar todas as alterações propostas, foram realizados testes com dados reais de produção na ferramenta de otimização CScheduler WP. Estes testes apresentaram resultados significativos para as áreas de negócio da ArcelorMittal Tubarão. Após a implantação da ferramenta, foi realizado o comissionamento da ferramenta para avaliação dos resultados. Concluímos que os resultados foram coerentes com os obtidos na fase de testes. A ferramenta está em uso para o planejamento e programação das máquinas de lingotamento contínuo da ArcelorMittal Tubarão.

\section{REFERÊNCIAS}

1 SLACK, Nigel; CHAMBERS, Stuart; JOHNSTON, Robert; CORREAA, Henrique Luiz. Administração da Produção. 2 ed. São Paulo: Atlas, 2002.

2 GUO, R.; CHIANG, D. M.; PAI, F. A WIP-based exception-management model for integrated circuit back-end production processes. The International Journal of Advanced Manufacturing Technology Vol. 33 pp. 1263-1274, 2006.

3 LINN, R. J.; WEI, Z.; LI, Z. An intelligent management system for technology management. Computers \& Industrial Engineering Vol.38, pp.397-412, 2000.

4 SLACK, N.; LEWIS, M. Estratégia de Operações. Porto Alegre: Bookman, 2009.

5 GAITHER, N.; FRAZIER, G. Administração da produção e operações. São Paulo: Cengage Learning, 2001.

6 TUBINO, Dalvio Ferrari. Manual de planejamento e controle da produção. 2 ed. São Paulo: Atlas,2000. 